\title{
CRYSTAL FIELD IN ErMg, ErPd AND RELATED COMPOUNDS
}

\author{
P. MORIN, J. PIERRE and D. SCHMITT \\ Laboratoire de Magnétisme, C.N.R.S., \\ 166-X, 38042 Grenoble Cedex, France \\ and \\ W. DREXEL \\ Institut Laue-Langevin, X56, 38042 Grenoble Cedex, France \\ (Reçu le 11 décembre 1975, accepté le 21 janvier 1976)
}

\begin{abstract}
Résumé. - Nous avons déterminé les paramètres de champ cristallin dans les composés cubiques ErMg et ErPd par spectroscopie neutronique, et par mesure de l'anisotropie magnétocristalline de ErMg. Nous analysons la variation des paramètres dans ces composés et les composés ErM isomorphes précédemment étudiés, en fonction de la valence de l'élément $\mathbf{M}$. L'évolution du paramètre $A_{4}\left\langle r^{4}\right\rangle$ semble refléter la localisation progressive des électrons d des métaux de transition alliés. Ce paramètre change de signe dans ErMg, où il devient positif. Le terme $A_{6}\left\langle r^{6}\right\rangle$ reste négatif et du même ordre de grandeur dans tous les composés; son origine est encore mal comprise.

Abstract. - The crystal field parameters have been determined in the cubic equiatomic compounds ErMg and ErPd by neutron spectroscopy, and by measuring the magnetocrystalline anisotropy of ErMg. The variation of the crystal field parameters, in these compounds and isomorphous ErM compounds previously studied, is analyzed as a function of the valency of $\mathbf{M}$. The behaviour of $A_{4}\left\langle r^{4}\right\rangle$ seems to reflect the progressive localization of d electrons in the alloyed transition metal. This parameter changes its sign in ErMg, where it turns positive. The $A_{6}\left\langle r^{6}\right\rangle$ term remains negative and of same order of magnitude in all compounds; its origin is still not understood.
\end{abstract}

1. Introduction. - Crystal field studies in rare earth intermetallic compounds have received much attention in the recent past [1]. As expected for metallic lattices, the point charge model often fails to give satisfactory accounts for the observed results. Moreover, the crystal field (CEF) parameters are sometimes found to vary drastically with the rare earth ion in a given series of compounds $[2,3]$. The present status of theory tries to clear up the contribution of the conduction electron distribution to the coulombic potential and to anisotropic exchange terms acting on the $4 \mathrm{f}$ shell [4-6].

In order to clarify the dependence of CEF parameters on the valency and size of ligand atoms and on the electronic structure of the compounds, we have undertaken a systematic determination of the crystal field in equiatomic compounds of the $\mathrm{CsCl}$ structure between rare earths $R$ and different metals $M$. In a preceding paper [7], referred to here as paper I, we reported our investigations by neutron spectroscopy on the erbium compounds with $\mathrm{Cu}, \mathrm{Ag}$ and $\mathrm{Zn}$. Some rhodium compounds were simultaneously studied by Chamard-Bois et al. [8]. In this paper, we describe new results for ErMg and ErPd, and we try to explain the variation of CEF parameters through different series. The comparison between ErMg and other compounds with transition metals should reveal the influence of $d$ electrons from these metals.

The study of erbium compounds is determined by the choice of a Kramers' ion, that reduces the number of possible transitions, and by the low magnetic interactions corresponding to ordering points below $20 \mathrm{~K}$. Some other compounds, particularly with holmium, were also investigated, and the results will be analyzed later.

2. Crystal structure and magnetic properties. 2.1 SAMPLE PREPARATION. - In the preceding study, we diluted erbium in the corresponding yttrium compound in order to reduce the magnetic interactions. In the case of palladium compounds, those with $\mathrm{Y}$ or La do not crystallize within the $\mathrm{CsCl}$ structure. Moreover, ErPd may be obtained in this phase only by quenching from the melt, that was obtained using a levitation furnace; otherwise it undergoes a 
phase change around $550^{\circ} \mathrm{C}$ [9], probably to a $\mathrm{FeB}$ like modification.

We have tested the crystal structure of our samples by X-rays at room temperature and by neutron diffraction at 4.2 and $100 \mathrm{~K}$. ErMg was found to be stable with a room temperature lattice parameter $a=3.748 \AA$ in agreement with a previous determination [10]. In ErPd, starting with the pure $\mathrm{CsCl}$ phase at room temperature $(a=3.445 \AA)$, we found a partial structural change on cooling to low temperatures. The percentage of $\mathrm{CsCl}$ phase at low temperatures is variable, and estimated to be $60-70 \%$ in our neutron spectroscopy experiments; the second phase having the FeB-type structure with lattice parameters $(a=7.23 \AA, b=4.25 \AA, c=5.58 \AA)$ in agreement with previous determinations on $\mathrm{TbPd}$ and HoPd [11].

2.2 Magnetic DATA. - We have performed a study of the magnetic properties on a single crystal of ErMg. The paramagnetic susceptibility follows a Curie-Weiss law with an effective moment $\mu_{\mathrm{p}}=9.60 \mu_{\mathrm{B}}$ and a paramagnetic temperature $\theta_{\mathrm{p}}=9 \mathrm{~K}$. The compound orders at $5.5 \mathrm{~K}$, but the magnetic structure is complex and has not yet been solved. The magnetization has been studied in fields up to $140 \mathrm{kOe}$, the results will be discussed in connection with the CEF level scheme in part 4.

The paramagnetic susceptibility of ErPd follows a Curie-Weiss law with $\theta_{\mathrm{p}}=11 \mathrm{~K}$ and an effective moment $\mu_{\mathrm{p}}=9.2 \mu_{\mathrm{B}}$. It orders with a spontaneous magnetization at $11 \mathrm{~K}$.

3. Neutron spectroscopy. - 3.1 EXPERIMENTAL METHODS. - The experiments were performed as described in paper I [7] on the IN7 time of flight spectrometer at the I.L.L. high flux reactor of Grenoble. However, the statistical chopper was replaced by a Fermi chopper, which gives a lower and smoother background. The incident energies ranged from 6.1 to $54.9 \mathrm{meV}$, depending on the different energy transfers investigated. The spectra are dominated by an intense elastic line, the width of which (full width at half maximum) never exceeded the instrumental resolution (about $2.0 \mathrm{meV}$ for an incident energy of $36.4 \mathrm{meV}$ ).

Spectra were usually taken at two different temperatures in the paramagnetic range for each compound. The lower temperature was chosen slightly above the ordering point in order to observe only excitation processes from the ground level without significant broadening of the levels due to short range order or dynamic-exchange fields [12].

The higher temperature was chosen high enough to populate excited levels and to observe new transitions by excitation or deexcitation processes.

The counting time varied from 20 to 60 hours depending on sample temperature and incident energy.
3.2 NEUTRON SPECTROSCOPY RESULTS. - The spectra have been analyzed within the theoretical approach derived by Trammell [13] for the magnetic crosssection :

$$
\begin{aligned}
\frac{\mathrm{d}^{2} \sigma}{\mathrm{d} \Omega \mathrm{d} E}= & C\left(\frac{1.91 e^{2}}{2 m c^{2}} g_{J}\right)^{2} F^{2}(\mathbf{Q}) \frac{k_{\mathrm{f}}}{k_{\mathrm{i}}} \\
& \sum_{n, m} \rho_{m}\left|\left\langle n\left|J_{\perp}\right| m\right\rangle\right|^{2} \delta\left(E_{n}-E_{m}-\hbar \omega\right)
\end{aligned}
$$

where $k_{\mathrm{i}}$ and $k_{\mathrm{f}}$ are the wave vector of incident and diffused neutron, $F(\mathbf{Q})$ the form factor, $J_{\perp}$ is the component of the total angular momentum perpendicular to the scattering vector $\mathbf{Q}$ and $\rho_{m}$ the thermal population of the initial state $|m\rangle$. The energy eigenvalues $E_{m}$ and $E_{n}$ [14] as well as the matrix elements [15] are computed by diagonalizing the cubic CEF Hamiltonian, taken according to Lea, Leask and Wolf's formalism [14] as :

$$
\mathcal{H}=W\left[x \frac{O_{4}}{F_{4}}+(1-|x|) \frac{O_{6}}{F_{6}}\right] .
$$

The level scheme for $\mathrm{Er}^{3+}$ ion is given in figure 1 as a function of $x$.

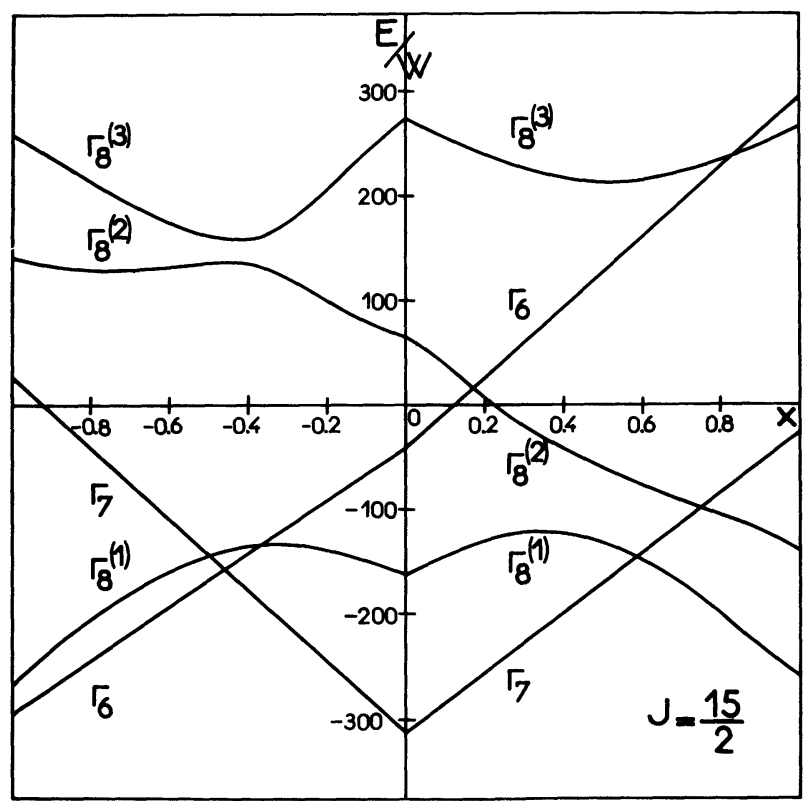

FIG. 1. - Level scheme for $J=15 / 2$ as a function of $x|14|$

As described in paper I, we used a fitting program to describe quantitatively the spectra assuming a gaussian shape for all structures. Besides the two CEF parameters, we take into account the instrumental resolution, the width of transitions, and the height of the elastic line. At high temperatures, a broad quasi-elastic contribution appears, but for clarity, we did not take it into account in the fits, which are drawn as solid lines in the figures.

Previous studies on non-magnetic YZn [7] and YRh [8] compounds have shown that phonon lines do not appear in our experimental conditions. The 
thermal variation of the observed structures also proves that they arise from crystal field transitions.

ErMg compound. - Spectra obtained on ErMg at $10 \mathrm{~K}$ and $53 \mathrm{~K}$ with an incident energy $E_{0}=36.24 \mathrm{meV}$ are given in figure 2. At $10 \mathrm{~K}$, we observe 3 transitions at 5.2, 8.5 and around $11.2 \mathrm{meV}$. These energy transfers and the transition intensities allow the determination of the level scheme without ambiguity, giving as parameters $W=-0.32 \pm 0.02 \mathrm{~K}$ and $x=-0.03 \pm 0.02$. The observed transitions connect the $\Gamma_{8}^{(3)}$ ground state to $\Gamma_{8}^{(2)}, \Gamma_{6}$ and $\Gamma_{8}^{(1)}$ levels respectively. The second temperature was chosen to populate the $\Gamma_{8}^{(2)}$ first excited level. The first peak is then broadened toward higher energy transfers due to the very intense $\Gamma_{8}^{(2)} \rightarrow \Gamma_{8}^{(1)}$ transition; and the deexcitation process from $\Gamma_{8}^{(2)}$ to $\Gamma_{8}^{(3)}$ is seen on the energy-gain side of the spectrum. The theoretical fit matches experimental data very well, as the ratio of CEF structures to elastic or quasi-elastic contributions is rather large.

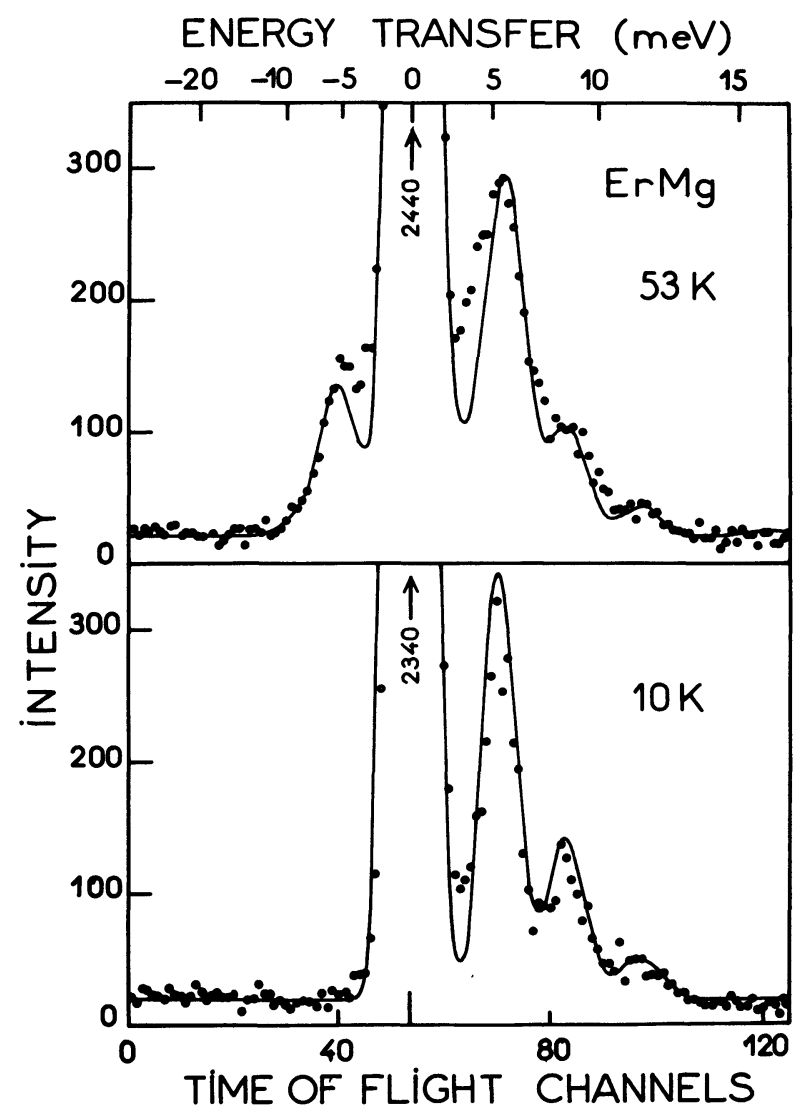

FIG. 2. - Time of flight spectra for ErMg at $10 \mathrm{~K}$ and $53 \mathrm{~K}$. The incident energy is $E_{0}=36.24 \mathrm{meV}$. The fit is drawn for $W=-0.32 \mathrm{~K}, x=-0.03$.

ErPd compound. - Several spectra were taken at different incident energies and temperatures ranging between 20 and $55 \mathrm{~K}$. On the spectrum taken at $22 \mathrm{~K}$ with $E_{0}=46.4 \mathrm{meV}$ (Fig. 3), two transitions are visible : one at $5 \mathrm{meV}(5.2 \mathrm{meV}$ from other spectra) and another large one around $15 \mathrm{meV}$. No

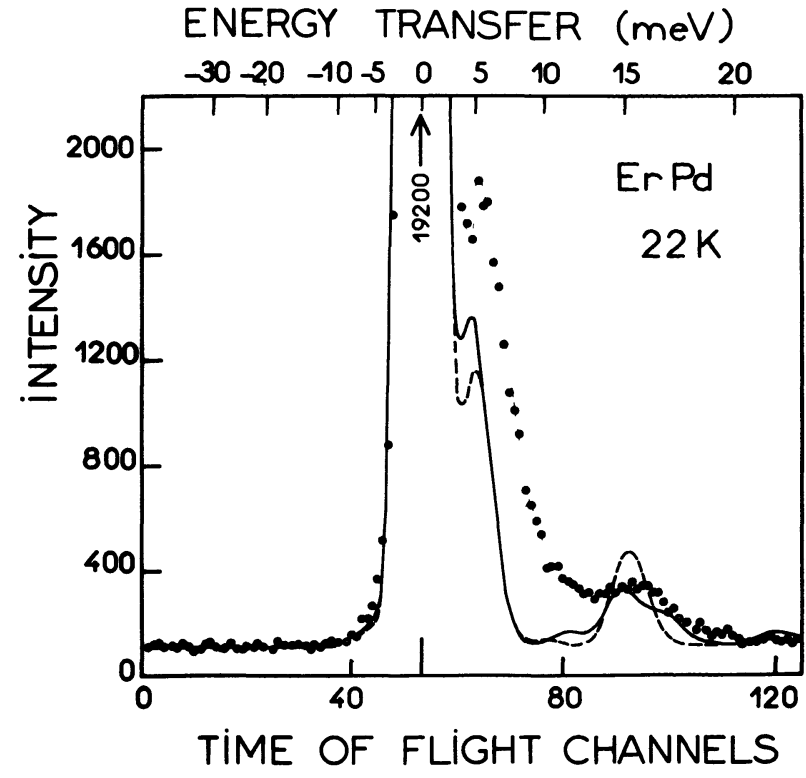

FIG. 3. - Time of flight spectrum for ErPd at $22 \mathrm{~K}$ with $E_{0}=46.4 \mathrm{meV}$. The fit in solid line corresponds to $W=-0.575 \mathrm{~K}$, $x=-0.22$; the dashed curve to $W=-0.65 \mathrm{~K}, x=+0.48$.

other lines were observed in different conditions, except the deexcitation process at $5 \mathrm{meV}$.

We must recall that we have a certain amount of FeB-like phase in our sample. However, the $\mathrm{CsCl}$ structure having a much higher symmetry is expected to give rise to fewer and more intense transitions, thus we try to interpret our diagram as mainly due to the $\mathrm{CsCl}$ phase.

With the aid of the energy transfers and transition intensities, we searched for a solution in the Lea, Leask and Wolf's level scheme diagram. Two sets of parameters give correct energy transfers : the first one $W=-0.575 \pm 0.03 \mathrm{~K}, x=-0.22 \pm 0.02$ leads to an intensity ratio in close agreement with the experimental one ; the second $W=-0.65 \pm 0.03 \mathrm{~K}$, $x=+0.48 \pm 0.02$ predicts a more intense $15 \mathrm{meV}$ transition than observed. However, due to the presence of the second phase, the two solutions are possible (Table I).

4. Magnetic anisotropy of ErMg. - We shall now analyze the magnetization data obtained for ErMg. As pointed out above, the magnetic structure of this compound is not ferromagnetic. However, due to the low ordering temperature $(5.5 \mathrm{~K})$ we expect that fields higher than $100 \mathrm{kOe}$ are sufficient to overcome antiferromagnetic interactions and magnetocrystalline energy, and to align the moments. This was verified a posteriori by calculating the moment from the determined level scheme. Thus the significant data are the magnetizations in high fields along the three principal axes.

Experimentally the magnetization is strongly anisotropic ; it reaches $7.72,7.04$ and $6.76 \mu_{\mathrm{B}}$ respectively along $\langle 111\rangle,\langle 110\rangle$ and $\langle 100\rangle$ directions under $140 \mathrm{kOe}$, and increases almost linearly in this 
TABLE I

Lattice parameter (room temperature), crystal field parameters and electronegativity differences (Pauling's scale)

Compound
$\operatorname{ErRh}\left({ }^{a}\right)$
$\operatorname{ErPd}\left({ }^{b}\right)$
$\operatorname{ErAg}\left({ }^{c}\right)$
$\operatorname{ErCu}\left({ }^{c}\right)$
$\operatorname{ErZn~}\left({ }^{c}\right)$
$\operatorname{ErMg}\left({ }^{b}\right)$
$\left(^{a}\right)$ Reference [8].
$\left(^{b}\right)$ Present work.
${ }_{(c)}$ Reference [7].

$a(\AA) \quad W(\mathrm{~K})$

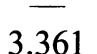

3.445

3.574

3.430

3.531

3.748

$-0.32$

\begin{tabular}{r}
$x$ \\
- \\
\hline 0.38 \\
-0.22 \\
0.48 \\
0.42 \\
0.34 \\
0.16 \\
-0.03
\end{tabular}
$A_{4}\left\langle r^{4}\right\rangle$
$+3.6$

$A_{6}\left\langle r^{6}\right\rangle$

$\Delta \Phi$
(K)
$(\mathrm{K})$

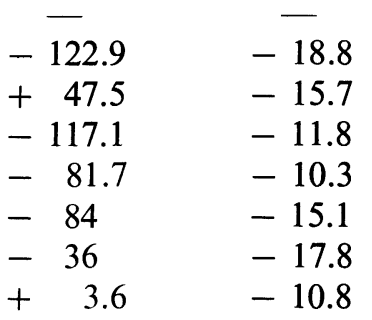

$\overline{1.0}$

1.0

0.7

0.7

0.4

0.16

0

range due to the wave function purification under field.

We have calculated the theoretical values by diagonalizing the whole Hamiltonian including the Zeeman term along the three axes. The best fit to magnetic data is obtained for $W=-0.4 \pm 0.05 \mathrm{~K}$, $x=-0.10 \pm 0.05$ (Table II), values in close agreement with neutron spectroscopy results in the paramagnetic range. In particular, the largest value of magnetization along $\langle 111\rangle$ axis implies that the fourth order CEF parameter $W x$ is positive, contrary to the case of ErZn where the magnetization was found largest along $\langle 100\rangle[16]$.

\section{TABLE II}

Moments observed in an internal field $H_{\mathrm{i}}=140 \mathrm{kOe}$, and calculated values for $W=-0.4 \mathrm{~K}, x=-0.1$

$\begin{array}{ccc}\begin{array}{c}\text { direction } \\ \text { of field }\end{array} & \mu_{\text {obs }}\left(\mu_{\mathbf{B}}\right) & \mu_{\text {calc }}\left(\mu_{\mathbf{B}}\right) \\ - & - & - \\ \langle 111\rangle & 7.72 & 7.60 \\ \langle 110\rangle & 7.04 & 7.07 \\ \langle 100\rangle & 6.76 & 6.78\end{array}$

5. Discussion of crystal field parameters. - We have tabulated in table I the values of the CEF parameters $W, x$,

$$
A_{4}^{0}\left\langle r^{4}\right\rangle=W x / \beta F_{4}
$$

and

$$
A_{6}^{0}\left\langle r^{6}\right\rangle=W(1-|x|) / \gamma F_{6}
$$

which have been determined in the present compounds, together with previous determinations on ErRh [8], $\mathrm{ErCu}, \mathrm{ErAg}$ and $\mathrm{ErZn} \mathrm{[7].} \mathrm{The} \mathrm{room} \mathrm{temperature}$ lattice parameters $(a)$ are also plotted, as well as electronegativity differences between constituents.

From a first analysis of the results, it seems likely that CEF parameters $A_{4}^{0}$ and $A_{6}^{0}$ for ErPd have same negative sign as in the neighbouring compounds ErRh and ErAg. This rules out the alternative solu- tion with $x<0$. With this assumption, the $A_{4}^{0}\left\langle r^{4}\right\rangle$ term is found to increase continuously from negative values with the number of outer electrons of alloyed metal through the sequence $\mathrm{Rh}, \mathrm{Pd}, \mathrm{Cu}, \mathrm{Ag}, \mathrm{Zn}, \mathrm{Mg}$, the sign being just reversed for $\mathrm{Mg}$ (Fig. $4 a$ ). The $A_{6}^{0}\left\langle r^{6}\right\rangle$ term is always found negative and of the same order of magnitude ; for isoelectronic compounds, it decreases with the lattice parameter $a$, but less rapidly than $a^{-7}$ as it should be in the point charge model.
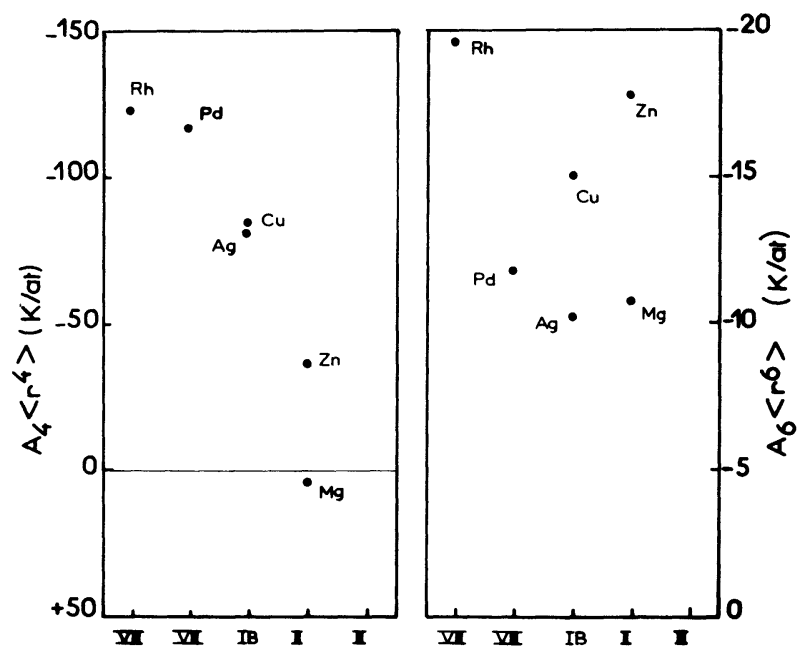

FIG. 4. - Variation of CEF parameters for ErM compounds.

As shown previously in paper I, the CEF parameters are much larger in these series than expected within a point charge model (except for $A_{4}^{0}\left\langle r^{4}\right\rangle$ in ErMg). Thus, as in most metallic systems, the effect of conduction electrons is not limited to the shielding of point charges.

.The different contributions to crystal field arise from [17] :

a) the effective charges borne by ligands,

b) the coulombic term from the anisotropic distribution of conduction electrons,

c) the anisotropic exchange term between $4 \mathrm{f}$ shell and the conduction band. 
From augmented-plane-wave (APW) calculations, the effective charges may be evaluated as :

$$
Z_{\text {i eff }}=Z_{\mathrm{i}}-n_{\mathrm{i}}+\Omega_{\mathrm{i}} \rho
$$

where $Z_{\mathrm{i}}$ is the ionic charge, $n_{\mathrm{i}}$ the number of conduction electrons within the APW atomic sphere, the volume of which is $\Omega_{\mathrm{i}}$, and $\rho$ is the uniform plane wave density between spheres. As an example, we give in table III the figures obtained through selfconsistent calculations for DyZn [18] and YMg [19] with the corresponding CEF contributions.

\section{TABLE III}

Effective charges from APW calculations and their contribution to CEF parameters

$\begin{array}{lcccc} & Z_{\text {eff }}(\mathrm{R}) & Z_{\text {eff }}(\mathrm{M}) & \begin{array}{c}A_{4}^{0}\left\langle r^{4}\right\rangle \\ (\mathrm{K})\end{array} & \begin{array}{c}A_{6}^{0}\left\langle r^{6}\right\rangle \\ (\mathrm{K})\end{array} \\ \text { DyZn } & 3.0 & - & - & - \\ \mathrm{YMg} & 2.84 & 1.7 & \sim 0 & -1 \\ & & 1.96 & 6.4 & -1\end{array}$

These contributions are rather small, due to the cancellation of terms from rare earth and metal $\mathbf{M}$ ligands.

The contribution of conduction electron distribution to the crystal field in rare earth metals has been investigated by several workers. Williams and Hirst [2] have evaluated the coulombic contribution of a $5 \mathrm{~d}$ virtual bound state for a rare earth diluted in noble metals. Chow [20] has shown that exchange effects lead to partial cancellation of the fourth order term in this case. Contributions from conduction electrons to the electric field gradients in rare earth metals have been calculated within the augmentedplane-wave (APW) formalism [4] or orthogonalizedplane-wave (OPW) approach [5]. The predominant contributions to $A_{2}^{0}\left\langle r^{2}\right\rangle$ and $A_{A}^{0}\left\langle r^{4}\right\rangle$ come from $\mathrm{d}$ electrons whereas $\mathrm{f}$ electrons also contribute to $A_{6}\left\langle r^{6}\right\rangle$. Eagles [21] has recently investigated the influence of $f-d$ interactions on the effective crystal field seen by the $4 \mathrm{f}$ shell.

We shall try to analyze our results with the help of band calculations performed on these compounds $[18,19]$. In cubic symmetry, the sign of $A_{4}^{0}\left\langle r^{4}\right\rangle$ is connected to the d-electrons character. $\mathrm{e}_{\mathrm{g}}$-type $(\mathrm{d} \gamma)$ wave functions pointing along fourfold axes lead to a positive coulombic contribution, $\mathrm{t}_{2 \mathrm{~g}}$-type $(\mathrm{d} \varepsilon)$ to a negative one.

In our compounds, we have to deal with two kinds of d-electrons, depending on their localization around $\mathrm{R}$ or $\mathrm{M}$ atoms. In $\mathrm{RCu}$ or $\mathrm{RZn}$ compounds, they are found [18] to form two rather well separated bands, whereas they are completely hybridized in $\mathrm{RRh}$ compounds.

i) A first band is built mainly from 3d (4d) electrons of the transition metal $\mathrm{M}$; it is absent in $\mathrm{RMg}$. These electrons become more and more localized on $\mathrm{M}$ from RRh to $\mathrm{RZn}$ as the band becomes nar- rower and lower in energy. The splitting of the band by crystal field, measured by the distance $\Gamma_{12}-\Gamma_{25}$, is of the same order of magnitude as the band width; however, the bottom of the band is mainly of $e_{\mathrm{g}}$-type and the top of $t_{2 \mathrm{~g}}$-type. As $\mathrm{e}_{\mathrm{g}}$ wave functions have lower energies and point along fourfold axes, we may expect that rare earth-transition metal bonding occurs through $t_{2 g}$ electrons. Thus the $t_{2 g}$ character induced on the rare earth should lead to a negative coulombic contribution to $A_{4}^{0}\left\langle r^{4}\right\rangle$. Following the sequence $\mathrm{Rh}, \mathrm{Pd}, \mathrm{Cu}, \mathrm{Ag}, \mathrm{Zn}$, the contribution from these electrons decreases as they become more localized around $M$.

ii) Meanwhile, a second $\mathrm{d}$ band arising mainly from the $5 \mathrm{~d}$ state of the rare earth is progressively filled from $\mathrm{Pd}$ to $\mathrm{Zn}$ or $\mathrm{Mg}$. This band is split almost completely by the crystal field term and only the $\mathrm{e}_{\mathrm{g}}$-subband is occupied; its own contribution to $A_{4}\left\langle r^{4}\right\rangle$ is positive increasing through the above sequence, which reinforces the preceding evolution. This second band is present only in ErMg and we therefore expect that the coulombic contribution to $A_{4}\left\langle r^{4}\right\rangle$ is positive.

Calculations of the exchange terms between $4 \mathrm{f}$ shell and the conduction band have just been started for such systems [22]. Although a large anisotropic exchange coupling with $d$ electrons is derived, no conclusions are yet available relative to the influence on CEF parameters. The importance of this term - or the importance of orthogonalization processes between $4 \mathrm{f}$ shell and the conduction band - is revealed by the rapid variation of CEF parameters with the rare earth ion in a given series (for instance $R Z n$ compounds [23]). But we are unable at present to take this term into account.

Bucher and Maita [24] correlate the variations of $A_{4}\left\langle r^{4}\right\rangle$ in $\mathrm{PrX}$ compounds of $\mathrm{NaCl}$ structure with the electro-negativity $\Phi$ of $\mathrm{X}$ element : the effective charge of the anion in these ionic compounds is shown to vary linearly with $\Phi$. We have given in table I the electronegativity differences $\Delta \Phi$ (Pauling's scale) between the rare earth and the alloyed metal. The experimental variations of $A_{4}^{0}\left\langle r^{4}\right\rangle$ follow rather well the variation of $\Delta \Phi$. This seems to reveal some significant electron transfer in our compounds : Miedema et al. [25] have pointed out that, in intermetallic compounds, the charge transfer $\Delta Z$ between elements was proportional to their electronegativity difference. However some difficulties appear when a careful analysis is followed through, the sign or amplitude of charge transfers given by band calculations or isomer shifts [26] do not agree with these theoretical predictions.

Turning now to the sixth order term, it is difficult to explain its large and rather constant order of magnitude. Effective charges on ligands were shown to give a small term. The contribution of conduction electrons within the central rare earth sphere should arise only from their $\mathrm{f}$ character, but APW calcula- 
tions [18] have shown a very small amount of f-like electrons, without evident correlations with the $A_{6}\left\langle r^{6}\right\rangle$ value. We may also expect that delocalized $\mathrm{d}$ electrons on transition metal ligands could give some contribution, but the large value encountered even in $\mathrm{RMg}$ seems to rule out a dominant influence from these electrons. Thus the origin of the sixth order term is still not understood.

6. Conclusion. - In the preceding sections, we have derived the CEF parameters in some isomorphous compounds, and shown their dependence on the valency and on the localization of d-type electrons of ligand atoms. The behaviour of the fourth order term is thus qualitatively understood.
A priori calculations for the crystal field parameters are now undertaken starting with the distribution of conduction electrons computed by the APW method. They should at least give the order of magnitude of the coulombic terms.

We have also restricted our investigations in this paper to one-ion terms in a cubic environment. This is only valid for dilute compounds or in the paramagnetic state. The magnetostriction observed for instance in $\mathrm{RZn}$ compounds [16], [23] reveals the influence of two-ion anisotropic exchange, arising for instance through the coupling of the $4 \mathrm{f}$ shell quadrupole with the conduction band [27]. Such terms must be taken into account for a proper understanding of the behaviour of concentrated systems.

\section{References}

[1] COOPER, B. R., in Magnetic properties of rare earth metals, ch. 2 (Ed. R. J. Elliott, Plenum Press) 1972. See also : Proc. of the Montreal conf. on Crystal Field in Metals and Alloys (Ed. R. A. B. Devine, Montreal) 1974.

[2] Williams, G., Hirst, L., Phys. Rev. 185 (1969) 407.

[3] Hфg, J., Touborg, P., Phys. Rev. $\mathrm{9}$ (1974) 2920. Phys. Rev. Lett. 33 (1974) 775.

[4] Das, K. C., Ray, D. K., Solid State Commun. 8 (1970) 2025.

[5] Devine, R. A. B., Dixon, J. M., Phys. Rev. B 7 (1973) 4902.

[6] Dixon, J. M., Duprée, R., J. Phys. F : Metal Phys. 3 (1973) 118.

[7] Morin, P., Pierre, J., Rossat-Mignod, J., Knorr, K., Drexel, W., Phys. Rev. B 9 (1974) 4932.

[8] Chamard-Bois, R., Rossat-Mignod, J., KnorR, K., DreXel, W., Solid State Commun. 13 (1973) 1549.

Chamard-Bois, R., Rossat-Mignod, J., Knorr, K., Drexel, W., Morin, P., Pierre, J., Proc. of the Montreal Conf. on Crystal Field in Metals and Alloys Ed. R. A. B. Devine, Montreal 1974.

[9] Löbich, O., Raub, E., J. Less Common Met. 30 (1973) 47.

[10] Buschow, K. H. J., J. Less Common Met. 33 (1973) 239.

[11] Inagawa, K., Watanabe, K., Kaneko, T., Ido, H., WataNabe, H., J. Phys. Soc. Japan 36 (1974) 1709.

[12] Furrer, A., Heer, H., Phys. Rev. Lett. 31 (1973) 1350.

[13] Trammell, G. T., Phys. Rev. 92 (1953) 1387.
[14] Lea, K. R., Leask, J. M., Wolf, W. P., J. Phys. Chem. Solids 23 (1962) 1381.

[15] Birgeneau, R. J., J. Phys. Chem. Solids 33 (1972) 59.

[16] Morin, P., Pierre, J., Phys. Stat. Sol. (a) 17 (1973) 479.

Aleonard, R., Morin, P., Pierre, J., Colloque Int. C.N.R.S., C 242, 39 (Ed. C.N.R.S., Paris) 1974.

[17] Freeman, A. J., in Magnetic Properties of Rare Earth Metals (Ed. R. J. Elliott, Plenum Press) 1972, Ch. 6, p. 325.

[18] Belakhovsky, M., Pierre, J., Ray, D. K., J. Phys. F : Metal Physics 5 (1975) 2274.

Belakhovsky, M., Ray, D. K., Phys. Rev. B 12 (1975) 3956.

[19] Schmitt, D., Pierre, J., Belakhovsky, M., J. Phys. F : Metal Phys. 6 (1976) under press.

[20] Chow, H. C., Phys. Rev. B 7 (1973) 3404.

[21] Eagles, D. M., Z. Phys. B 21 (1975) 171

[22] Ray, D. K., Solid State Commun. 15 (1974) 1471.

[23] Morin, P., Pierre, J., Phys. Stat. Sol. (a) 30 (1975) 549.

[24] Bucher, E., Maita, J. P., Solid State Commun. 13 (1973) 215.

[25] Miedema, A. R., De Boer, F. R., DE Chatel, P. F., J. Phys. F : Metal Phys. 3 (1973) 1558.

[26] Belakhovsky, M., RaY, D. K., Solid State Commun. 17 (1975) 349.

[27] Friederich, A., Fert, A., Phys. Rev. Lett. 33 (1974) 1214.

Fert, A., Friederich, A., Proc. of Int. Magnetism Conf. of San Francisco (1974). 